\title{
Occult tumor metastasis and the prognostic value of sentinel lymph nodes in rectal cancer
}

\author{
XIUTIAN GUO ${ }^{1,3}$, CUN WANG ${ }^{2,3}$, XIAO-GANG SHEN ${ }^{2}$, \\ SI-QIN DING ${ }^{2}$, YONG-YANG YU ${ }^{2}$ and ZONG-GUANG ZHOU ${ }^{2}$ \\ ${ }^{1}$ Department of Anal and Rectal Surgery, Longhua Hospital, Shanghai University of TCM, Shanghai 200032; \\ ${ }^{2}$ Department of Gastrointestinal Surgery and Institute of Digestive Surgery, \\ West China Hospital, Sichuan University, Chengdu 610041, P.R. China; \\ ${ }^{3}$ Department of Colorectal Surgery, Cleveland Clinic, Cleveland, OH 44195, USA
}

Received July 5, 2011; Accepted November 2, 2011

DOI: $10.3892 / \mathrm{ol} .2011 .490$

\begin{abstract}
The aim of the present study to evaluate the application of sentinel lymph node (SLN) pathology in rectal cancer using ex vivo mapping and to investigate the incidence and prognostic value of occult SLN metastasis in routine node-negative specimens. Specimens $(n=117)$ of rectal cancer were examined using a combination of routine pathology and ex vivo SLN mapping. The inspected SLNs were further treated with immunohistochemical staining for occult cancer foci. The log-rank test was used to assess survival. SLNs were examined in 112 of the included specimens with a total number of 212, resulting in an identification rate of $95.7 \%$ $(112 / 117)$. The status of SLNs accurately reflected N stage in $93.8 \%(105 / 112)$ of cases and the sensitivity was $75.6 \%$ (31/41) in detecting nodal metastasis. The accuracy of SLN pathology decreased in cancers of more advanced TNM stages $(\mathrm{P}=0.001)$. In 74 cases with routine node-negative (N0) disease, SLN micrometastasis (MIC) and isolated tumor cells (ITC) were examined in 9 and 4 cases, while the remaining 61 were regarded as negative (NEG). The log-rank test revealed poorer disease-free and overall survival of the MIC group compared with the NEG group. However, the findings from the ITC group were not significant compared with those from the MIC and NEG groups. In conclusion, ex vivo SLN pathology is practical and accurate in rectal cancer; however, its utility is limited in cases of advanced disease. Immunohistochemically detected SLN micrometastasis in node-negative cases is therefore a predictor of poorer outcome, and may therefore be listed as a marker for adjuvant therapy.
\end{abstract}

Correspondence to: Dr Cun Wang, Department of Gastrointestinal Surgery, West China Hospital, Sichuan University, Chengdu 610041, P.R. China

E-mail: c.wang.sf@gmail.com

Key words: rectal cancer, sentinel, lymph node, micrometastasis, prognosis

\section{Introduction}

Surgery remains the first choice of treatment for patients with rectal cancer and prognosis mainly depends on the stage of disease at the time of surgery (1). At present, the most valuable staging and prognostic indicator is lymph node status, i.e., whether the lymph nodes are involved in cancer or not $(2,3)$. Therefore, it is widely accepted that examination of a sufficient number of lymph nodes is essential for accurate staging, thus aiding prescription for adjuvant therapy $(4,5)$.

However, examination of the sentinel lymph node (SLN), defined as the node with the highest incidence of metastasis, was reported as being capable of reducing the pathological workload while maintaining diagnostic accuracy. If the results of SLN examination in breast cancer and melanoma could be duplicated in rectal cancer the effort and cost of harvesting an adequate number of nodes would no longer be necessary $(6,7)$. The present study therefore aimed to evaluate the application of SLN pathology in rectal cancer by mapping with patent blue $\mathrm{V}$ dye.

In addition, recurrence has been observed in those patients presenting with primarily node-negative disease, indicating that routine pathological examination may fail to detect certain occult tumor metastases $(8,9)$. Identification of these foci may aid the subdivision of node-negative patients for subsequent treatment to prevent relapse (10). Moreover, although the SLN of colorectal cancer has been discussed in several respects by existing studies, examination of the incidence and prognosis of occult cancer foci is still lacking from the literature. Therefore, we also examined micrometastasis and isolated tumor cells of SLN by investigating their prognostic value with the aim of providing substantial data for clinical practice.

\section{Materials and methods}

Patients. A total of 126 consecutive patients with biopsyproven rectal cancer who underwent radical resection at the Department of Gastrointestinal Surgery, West China Hospital, Sichuan University, China, between September 2004 and June 2005 were included in this study. Exclusion 
criteria included invasion of other organs and multi-original diseases. Of these 126 patients, 3 were lost during follow-up, 4 succumbed to diseases other than rectal cancer and 2 patients refused routine postoperative testing. After exclusion of these patients, the remaining 117 patients were assessed.

The study comprised 69 male and 48 female patients with a median age of 58 years (range 24-89). No patient received preoperative chemotherapy or radiotherapy.

The study was approved by the institutional review board of West China Hospital, Sichuan University. A consent form was signed by all included patients at the time of entering the study.

Surgical technique. Standard surgical resection with lymphadenectomy was performed. The ex vivo SLN mapping was carried out within 5 min of specimen removal according to published studies $(11,12)$. The rectum was initially incised longitudinally along the anterior wall. A subserosal peritumoral injection of $0.5-2 \mathrm{ml}$ of patent blue $\mathrm{V}$ dye (Ben Venue Labs, Bedford, OH, USA) was administered. The specimen was massaged as necessary to enhance lymphatic flow. The mesorectum was then examined for the first 1-4 blue-stained nodes by transillumination. The inspected blue nodes were identified as SLN and dissected for further treatment.

Specimen preparation. After sentinel lymph node biopsy, the remaining specimens were sent to routine pathology for detailed histologic findings. The lymph nodes harvested, including the SLN, were marked and embedded. Serial sections sampled randomly from representative levels were then subjected to hematoxylin and eosin (H\&E)staining. Except for this routine $H \& E$ procedure, same-level sections of the SLN-containing blocks were further analyzed by immunohistochemical (IHC) staining with three monoclonal antibodies, including anti-CK 20 (Dako, Glostrup, Denmark), anti-CK 8 (Thermo, Waltham, MA, USA) and anti-CEA (Dako). Substitution of the primary monoclonal antibody with phosphate-buffered solution was used as a negative control and previously confirmed rectal adenocarcinoma tissue served as a positive control.

According to the AJCC guidelines, neoplastic foci of sizes $0.2-2 \mathrm{~mm}$ were defined as micrometastasis (MIC), whereas those $<0.2 \mathrm{~mm}$ were defined as isolated tumor cells (ITCs) (13). Slides were reviewed independently by two experienced pathologists and reconfirmed by their senior.

Follow-up. Follow-up was performed by a combination of outpatient visits, and by letter and telephone. Patients generally underwent physical examination (including digital examination) and blood testing (including measurement of CEA level) at 3-month intervals, chest radiography and ultrasonography of the liver at 6-month intervals, and annual surveillance colonoscopy. CT or magnetic resonance imaging (MRI) was performed when tumor relapse was suspected.

No postoperative mortality was noted. The median time of follow-up was 57 months (range 11-62).

Statistical analysis. Data were analyzed using the SPSS 13.0 package. The Chi-square test was used to determine statistical significance. The independent sample t-test was used to compare the age and tumor size of the studied groups. Postoperative survival was calculated using the Kaplan-Meier method with a log-rank test. $\mathrm{P}<0.05$ was considered to be statistically significant.

\section{Results}

The surgery was regarded as curative by the surgeons. Donuts included in the stapler device were also examined microscopically and were histologically free of cancer. The characteristics of studied patients are shown in Table I.

Sentinel lymph node examination. A total of 212 (average 1.9, range 1-4) SLN were examined from the $112(112 / 117,95.7 \%)$ included specimens. With regard to the remaining 5 specimens with no SLN sampled, 1, 1 and 3 were in the TNM stage I, II and III groups, respectively. Thus, the identification rate of SLN was $96.0 \%(24 / 25)$ for TNM stage I disease and $98.0 \%(50 / 51)$ and $92.7 \%$ (38/41) for stages II and III, respectively. Moreover, 7 cases with TNM stage III disease were recorded as false-negatives since their SLN examinations were negative for cancer. Therefore, the accuracy of SLN pathology was $93.8 \%(105 / 112)$ in this study. Since 41 cases with nodal metastasis (TNM stage III) were included in this study, the sensitivity of SLN mapping was $75.6 \%$ (31/41) after subtracting the 7 false-negative and 3 unsampled cases.

Concerning the association between variables, the accuracy of SLN pathology decreased in cancers of more advanced TNM stages $(\mathrm{P}=0.001)$. However, no significant difference in accuracy was observed in patients of various genders $(\mathrm{P}=0.403)$, ages $(\mathrm{P}=0.550)$, primary tumor locations $(\mathrm{P}=0.699)$ and differentiations $(\mathrm{P}=0.457)$ (Table $\mathrm{I})$.

Micrometastasis and isolated tumor cells. Sentinel lymph nodes of 24 TNM stage I and 50 stage II specimens were examined in this study, and were termed node-negative (NO) cases. Further immunohistochemical analysis revealed that 9 cases harbored MIC in their SLN, while another 4 cases contained ITCs. The remaining 61 cases were regarded as negative (NEG).

Follow-up. During the follow-up period, 14 (14/74, 18.9\%) cases of recurrence were observed in the 74 cases. The majority of the recurrence $(8 / 14,57.1 \%)$ occurred within 3 years of surgery with a range of 11-51 months. The log-rank test revealed that patients with SLN micrometastasis $(\mathrm{P}=0.016)$ suffered significantly poorer disease-free survival (DFS) compared to those without SLN involvement (Fig. 1). For the group with ITC, the difference in the DFS rate was not significant compared with the MIC and NEG groups.

Mortality. Nine $(9 / 74,12.2 \%)$ deaths were recorded for the three groups mentioned above. Of the 9 patients, 6 were from the MIC group and 3 were from the NEG group. Although the majority $(6 / 9,66.6 \%)$ of the deaths occurred within 3 years, 3 occurred in the fifth year. The difference in overall survival rates between the MIC and NEG groups was significant $(\mathrm{P}=0.026)$. However, the results obtained from the ITC group were not significant when compared with those of the remaining 2 groups (Fig. 2). 
Table I. The accuracy of SLN pathology and its correlation with clinicopathological characteristics of the studied patients.

\begin{tabular}{lrrrr}
\hline & Total & Correct $^{\mathrm{a}}$ & Incorrect $^{\mathrm{b}}$ & P-value \\
\hline & 117 & 105 & 12 & \\
Gender & & & & \\
Male & 69 & 61 & 8 & 0.403 \\
Female & 48 & 44 & 4 & \\
Age (years) & & & & \\
$\quad$ Median & 58 & 58 & 56 & 0.550 \\
Range & $24-89$ & $24-89$ & $33-79$ & \\
Tumor distance to & & & & \\
anal verge (cm) & & & & \\
$\quad$ Median & 7 & 7 & 6 & 0.699 \\
Range & $1-16$ & $1-16$ & $3-13$ & \\
Tumor & & & & \\
differentiation & & & & \\
$\quad$ Well & 9 & 9 & 0 & 0.457 \\
Moderate & 72 & 65 & 7 & \\
$\quad$ Poor & 36 & 31 & 5 & \\
TNM stage & & & & \\
I & 25 & 24 & 1 & 0.001 \\
II & 51 & 50 & 1 & \\
III & 41 & 31 & 10 & \\
\hline
\end{tabular}

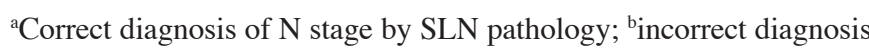
of N stage by SLN pathology or no SLN identified by ex vivo mapping.

\section{Discussion}

Lymph node metastasis is the main means by which rectal cancer spreads. In their study, Bilimoria et al proposed that postoperative pathological examination should involve all possible lymph nodes to aid personalized treatment (14). In a previous study, we increased the number of lymph nodes examined by combining the large tissue slice technique with tissue microarray (15). However, this detailed approach is not practical for daily pathologic application. Moreover, as reported recently, the harvested number of lymph nodes is a highly dependent variable correlating to a number of factors, including surgery, radiology and pathology $(16,17)$.

According to studies conducted on breast cancer and melanoma, SLN bears the highest incidence of metastasis $(18,19)$. Thus, its status potentially indicates the actual stage of disease. If this theory remains valid in rectal cancer, the associated cost and labor used in maximizing the harvested number of lymph nodes may no longer be required. Potential advantages of the ex vivo mapping technique over an in vivo procedure include exemption from tumor cell shedding and avoidance of adverse dye reactions (20).

Results of this study have shown an ex vivo sentinel lymph node mapping in rectal cancer with an identification rate of $95.7 \%$, an accuracy of $92.9 \%$ and a sensitivity of $75.6 \%$, which were in accordance with other studies $(20,21)$. Thus, an ex vivo SLN pathology may be considered a practical and

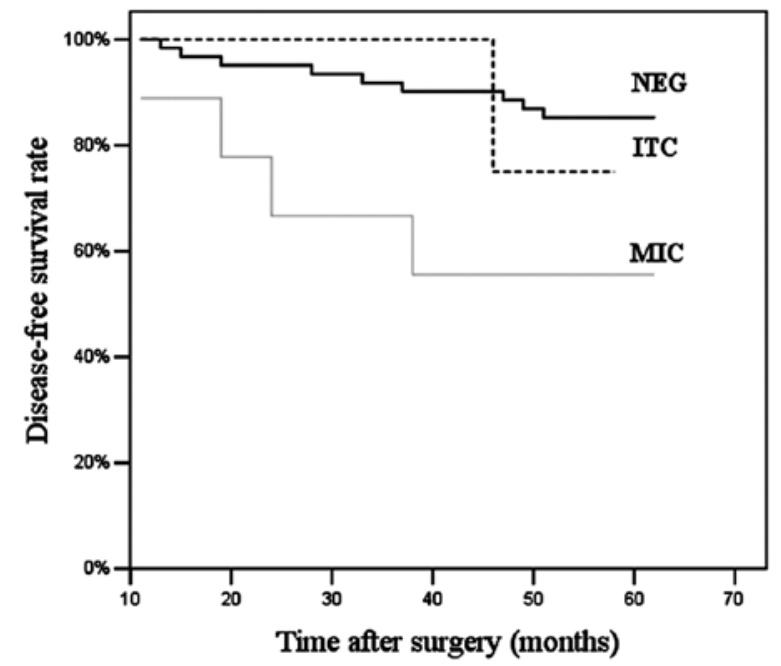

Figure 1. Disease-free survival curves of conventional N0 patients with different SLN status: NEG vs. MIC, $\mathrm{P}=0.016$; NEG vs. ITC, $\mathrm{P}=0.606$; ITC vs. MIC, $\mathrm{P}=0.429$ (log-rank test).

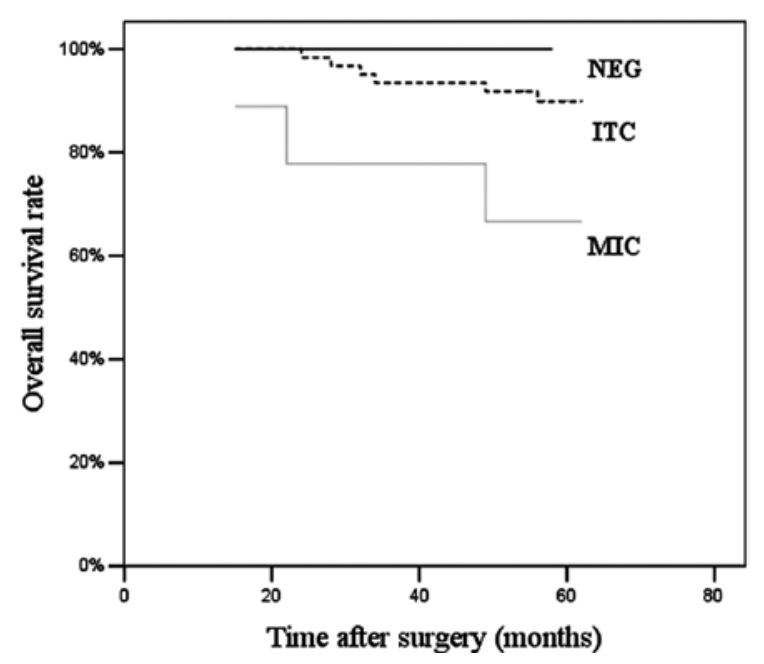

Figure 2. Overall survival curves of conventional N0 patients with different SLN status. NEG vs. MIC, $\mathrm{P}=0.025$; NEG vs. ITC, $\mathrm{P}=0.526$; ITC vs. MIC, $\mathrm{P}=0.219$ (log-rank test). NEG, patients with no tumor involvement of their sentinel lymph node $(n=61)$; ITC, patients with isolated tumor cells in their sentinel lymph node $(n=4)$; MIC, patients with micrometastasis in their sentinel lymph node $(\mathrm{n}=9)$.

reliable technique in rectal cancer. In addition, we have also identified an association between the decreased accuracy of SLN pathology and the advanced stages of disease, a phenomenon seldom mentioned by contemporaries. The results may be explained by the anatomic theory that clusters of cancer occlude the principal lymphatic drainage in infiltrating and spreading disease. Thus, a limitation of the SLN technique may be its inability to detect the small number of cases with aberrant lymphatic flow.

Although the majority of patients with node-negative rectal cancer are potentially curable by surgery alone, $20-30 \%$ of the patients succumb to local recurrence or distant metastasis (8-10). This occurrence may be explained by the dissemination of tumors into the lymphatic system, which cannot be detected by routine pathology. To investigate this possi- 
bility, we examined the micrometastasis and isolated tumor cells in SLNs in routine node-negative specimens. Although radical surgery of rectal cancer is performed using peri-fascial resection of the rectum and mesorectum that would not be guided by SLN mapping, detailed information regarding SLN may facilitate nodal stage subdivision and patient selection for further adjuvant therapy.

However, despite a focus on micrometastasis in numerous studies, the prognostic value of its occurrence remains to be determined. In this study, we performed an immunohistochemical analysis with three monoclonal antibodies for more accurate judgment of morphological correctness and preclusion of false-positive reactions. Therefore, unlike studies using reverse transcription-polymerase chain reaction (RT-PCR), the micrometastasis and isolated tumor cells observed in this study may accurately reflect the actual presence of tumor spread $(22,23)$.

Compared with patients without SLN involvement, we observed significantly poorer disease-free (55.6 vs. 85.2\%, $\mathrm{P}=0.016)$ and overall (66.7 vs. $90.2 \%, \mathrm{P}=0.025)$ survival rates for patients with SLN micrometastasis. This result indicates that adjuvant therapy should be recommended for these patients to prevent relapse. However, a comparison of recurrence and survival rates between the ITC and NEG groups was not significant. A larger cohort and longer follow-up may therefore be required to clarify the prognostic value of the isolated tumor cells observed.

In conclusion, ex vivo SLN mapping is a practical and accurate technique in the pathological examination of rectal cancer. However, its benefits are limited in cases of advanced disease with aberrant lymphatic flow. Immunohistochemically detected SNL micrometastasis in node-negative rectal cancer indicates poorer disease-free and overall survival and may therefore be regarded as a marker in patient selection for adjuvant therapy.

\section{Acknowledgements}

This study was supported by foundation for the author of national excellent doctoral dissertation of P.R. China (FANEDD 2007B66) and by the National Science Foundation of China (NSFC 30801331).

\section{References}

1. Nelson H, Petrelli N, Carlin A, Couture J, Fleshman J, Guillem J, Miedema B, Ota D and Sargent D: Guidelines 2000 for colon and rectal cancer surgery. J Natl Cancer 93: 583-596, 2001

2. Jemal A, Siegel R, Ward E, Murray T, Xu J and Thun MJ: Cancer statistics. CA Cancer J Clin 57: 43-66, 2007.

3. Bruch HP, Schwandner O, Schiedeck TH and Roblick UJ: Actual standards and controversies on operative technique and lymphnode dissection in colorectal cancer. Langenbecks Arch Surg 384: 167-175, 1999.

4. Joseph NE, Sigurdson ER, Hanlon AL, Wang H, Mayer RJ, MacDonald JS, Catalano PJ and Haller DG: Accuracy of determining nodal negativity in colorectal cancer on the basis of the number of nodes retrieved on resection. Ann Surg Oncol 10: 213-218, 2003.

5. Gill S, Loprinzi CL, Sargent DJ, Alberts SR, Haller DG, Benedetti J, Francini G, Shepherd LE, Francois Seitz J, Labianca R, et al: Pooled analysis of fluorouracil-based adjuvant therapy for stage II and III colon cancer: who benefits and by how much? J Clin Oncol 22: 1797-1806, 2004.
6. Morton DL, Duan-Ren W, Wong JH, Economou JS, Cagle LA, Storm FK, Foshag LJ and Cochran AJ: Technical details of intraoperative lymphatic mapping for early stage melanoma. Arch Surg 127: 392-399, 1992.

7. Hill AD, Mann GB, Borgen PI and Cody HS III: Sentinel lymphatic mapping in breast cancer. J Am Coll Surg 188: 545-549, 1999.

8. Faerden AE, Sjo OH, Bukholm IR, Andersen SN, Svindland A Nesbakken A and Bakka A: Lymph node micrometastases and isolated tumor cells influence survival in stage I and II colon cancer. Dis Colon Rectum 54: 200-206, 2011.

9. Bilchik AJ, Hoon DS, Saha S, Turner RR, Wiese D, DiNome M, Koyanagi K, McCarter M, Shen P, Iddings D, et al: Prognostic impact of micrometastases in colon cancer: interim results of a prospective multicenter trial. Ann Surg 246: 568-575, 2007.

10. Belt EJ, van Stijn MF, Bril H, De Lange-De Klerk ES, Meijer GA, Meijer S and Stockmann HB: Lymph node negative colorectal cancers with isolated tumor deposits should be classified and treated as stage III. Ann Surg Oncol 17: 3203-3211, 2010.

11. Saha S, Wiese D, Badin J, Beutler T, Nora D, Ganatra BK, Desai D, Kaushal S, Nagaraju M, Arora M and Singh T: Technical details of sentinel lymph node mapping in colorectal cancer and its impact on staging. Ann Surg Oncol 7: 120-124, 2000.

12. Wong JH, Steinemann S, Calderia C, Bowles J and Namiki T: Ex vivo sentinel node mapping in carcinoma of the colon and rectum. Ann Surg 233: 515-521, 2001.

13. Colon and rectum. In: AJCC Cancer Staging Manual, 6th ed. Greene FL, Page DL and Fleming ID (eds) Springer, New York, pp143-164, 2002.

14. Bilimoria KY, Bentrem DJ, Stewart AK, Talamonti MS, Winchester DP, Russell TR and Ko CY: Lymph node evaluation as a colon cancer quality measure: a national hospital report card. J Natl Cancer Inst 100: 1310-1317, 2008.

15. Wang C, Zhou ZG, Wang Z, Li L, Zheng YC, Zhao GP, Chen DY and Liu WP: Mesorectal spread and micrometastasis of rectal cancer studied with large slice technique and tissue microarray. J Surg Oncol 91: 167-172, 2005.

16. Mekenkamp LJ, van Krieken JH, Marijnen CA, van de Velde CJ and Nagtegaal ID: Lymph node retrieval in rectal cancer is dependent on many factors - the role of the tumor, the patient, the surgeon, the radiotherapist, and the pathologist. Am J Surg Pathol 33: 1547-1553, 2009.

17. Baxter NN, Virnig DJ, Rothenberger DA, Morris AM, Jessurun J and Virnig BA: Lymph node evaluation in colorectal cancer patients: a population-based study. J Natl Cancer Inst 97: 219-225, 2005.

18. Mulsow J, Winter DC, O'Keane JC and O'Connell PR: Sentinel lymph node mapping in colorectal cancer. Br J Surg 90: 659-567, 2003.

19. Smith FM, Coffey JC, Khasri NM, Walsh MF, Parfrey N, Gaffney E, Stephens R, Kennedy MJ, Kirwan W and Redmond HP: Sentinel nodes are identifiable in formalin-fixed specimens after surgeon-performed ex vivo sentinel lymph node mapping in colorectal cancer. Ann Surg Oncol 12: 504-509, 2005.

20. De Haas RJ, Wicherts DA, Hobbelink MG, Borel Rinkes IH, Schipper ME, van der Zee JA and van Hillegersberg R: Sentinel lymph node mapping in colon cancer: current status. Ann Surg Oncol 14: 1070-1080, 2007.

21. Bilchik AJ, Saha S, Wiese D, Stonecypher JA, Wood TF, Sostrin S, Turner RR, Wang HJ, Morton DL and Hoon DS: Molecular staging of early colon cancer on the basis of sentinel node analysis: a multicenter phase II trial. J Clin Oncol 19: 1128-1136, 2001

22. Kelder W, Braat A, van den Berg A, Platteel I, Hollema H, Groen $\mathrm{H}$ and Plukker J: Value of RT-PCR analysis of sentinel nodes in determining the pathological nodal status in colon cancer. Anticancer Res 27: 2855-2859, 2007.

23. Doekhie FS, Kuppen PJ, Peeters KC, Mesker WE, van Soest RA, Morreau H, van de Velde CJ, Tanke HJ and Tollenaar RA: Prognostic relevance of occult tumour cells in lymph nodes in colorectal cancer. Eur J Surg Oncol 32: 253-258, 2006. 